\title{
IDEALS IN THE SECOND CONJUGATE ALGEBRA OF A GROUP ALGEBRA
}

\author{
PAUL CIVIN
}

\section{Introduction.}

Let (SS denote an infinite locally compact abelian group, and let $L$ (SS) be its group algebra. The second conjugate space $L^{* *((S)}$ can be made into an algebra by the use of Arens multiplication [1] [2]. The algebra which is so obtained has been shown by Civin and Yood [6] to be not commutative and not semisimple. The object of the present work is to exhibit some of the properties of the maximal modular left ideals of $L^{* *}\left(\right.$ SS), or equivalently since $L^{* *}($ (S) $)$ has a right identity, of the maximal left ideals of $L^{* *((S)) . ~}$

Let $\pi$ denote the natural embedding of $L\left((\mathfrak{S )})\right.$ into $L^{* *}((\mathfrak{S )})$. In $\S 2$ it is shown that any maximal left ideal $\mathfrak{M}$ of $L^{* *}(\mathfrak{S})$ either satisfies $\mathfrak{M} \supset$ $\pi L(\mathfrak{S})$ or there is a unique character $\gamma \in \hat{\mathscr{S}}$ such that $\mathfrak{M} \cap \pi L(\mathfrak{S})$ is the injection into $L^{* *((S))}$ of the maximal ideal of $L(\mathbb{S})$ corresponding to $\gamma$. The stated phenomenon occurs despite the fact, also shown in $\S 2$, that $\pi L(\mathfrak{B S})$ is not an ideal of $L^{* *(G)}$ if $(\mathfrak{S}$ is not compact. In $\S 3$ it is shown that each of the various types of maximal left ideals, the possibility for whose existence arises from the results of $\S 2$, indeed exists. It is also shown in $\S 3$ that when $\mathscr{B S}$ is not compact there exist multiplicative linear functionals on $L^{* *}($ (S) $)$ which do not correspond to characters of (S). Section 4 deals with the relation of the behavior of a subspace of $L^{* *}(\mathfrak{B S})$ with respect to translation invariance to the question of whether the subspace is a left or right ideal. In $\S 5$ some results are obtained relating to the linear space annihilators of maximal left ideals. As a corollary, it is deduced that the only $w^{*}$-closed maximal left ideals are those determined by a character of the group.

It should be noted that the choice of the maximal modular left ideal as the subject of investigation is not an arbitrary one. The presence in $L^{* *}($ (S) $)$ of a right identity, which, for (S) not discrete, is not a left identity, makes the above ideals more accessible than the corresponding right ideals.

Received June 1, 1962.

This research was supported by the U. S. National Science Foundation, grant NSF-G-14, 111.

Math. Scand. $11-11$ 


\section{Maximal left ideals.}

Let $\mathfrak{M}_{* \gamma}$ denote the maximal ideal in $L(\mathfrak{S})$ which corresponds to the character $\gamma \in \hat{\mathbb{S}}$, that is,

$$
\mathfrak{M}_{* \gamma}=\left\{x \in L(\mathfrak{S}) \mid \int_{\mathscr{G}} \bar{\gamma}(t) x(t) d t=0\right\} .
$$

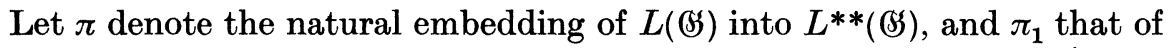

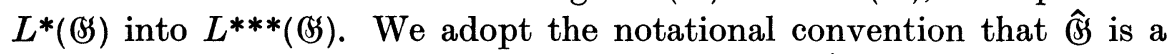
subset of $L^{*}(\mathfrak{S})$. Thus we may also write for $\gamma \in \hat{\mathfrak{S}}$,

$$
\mathfrak{M}_{* \gamma}=\{x \in L(\mathbb{S}) \mid \bar{\gamma}(x)=0\} .
$$

Since $\bar{\gamma}$ is a multiplicative linear functional on $L(\mathbb{S})$ if $\gamma \in \hat{\mathbb{S}}$, it follows

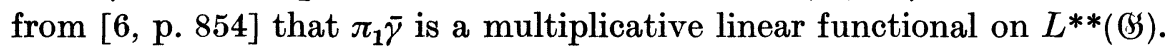
Consequently

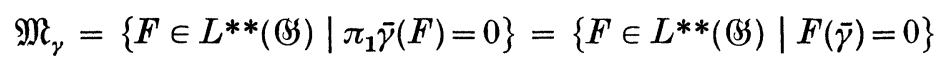

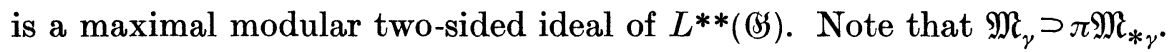
In fact it follows from [6, p. 865] that $\mathfrak{M}_{\gamma}$ is the $w^{*}$-closure of $\pi \mathfrak{M}_{* \gamma^{*}}$.

The above notation will be used throughout the sequel, as well as the symbol $\mathfrak{M}$ for an arbitrary maximal modular left ideal in $L^{* *}(\mathbb{G S})$. As noted in the Introduction, the latter is also a maximal left ideal in $L^{* *}(\mathbb{G})$ since $L^{* *}(\mathfrak{G})$ has a right identity [6, p. 855]. The symbol $E$ will be used for an arbitrary, but fixed right identity. Some further notation, however, is needed for the present. For $\theta$ a proper open set in $\hat{B}$, let

$$
S_{\theta}=\{x \in L(\mathfrak{H}) \mid \bar{\mu}(x)=0 \text { for all } \bar{\mu} \notin \theta\} .
$$

Also for $\gamma \in \hat{\mathfrak{S}}$, let

$$
T(\gamma)=\left\{\begin{array}{l|l}
x \in L(\mathbb{S}) & \begin{array}{l}
\text { There exist } \theta \text { open in } \hat{\mathbb{S}}, \\
\gamma \in \theta, \text { and } \bar{\mu}(x)=0 \text { for all } \bar{\mu} \in \theta
\end{array}
\end{array}\right\}
$$

Clearly, $T(\gamma)$ is an ideal in $L(\mathfrak{S})$ and $S_{0}$ is a closed ideal in $L(\mathscr{S})$.

Lemma 2.1. If $\mathfrak{M} \ngtr \pi T(\gamma), \gamma \in \hat{\mathfrak{S}}$, then there exists an open set $\theta$ in $\hat{\mathfrak{S}}$, $\gamma \in \theta$, and $\mathfrak{M} \supset \pi S_{\theta}$.

Proof. Suppose $\mathfrak{M} \ngtr \pi T(\gamma)$. Let $x \in T(\gamma)$, and $\pi x \notin \mathfrak{M}$. Then there exists an open set $\theta$ in $\hat{\mathfrak{S}}, \gamma \in \theta$, and for $\mu \in \theta, \bar{\mu}(x)=0$. Since $L^{* *}(\mathbb{S})$ has a right identity $E$, and $\pi L(\mathfrak{G})$ is central $\left[6\right.$, p. 855] in $L^{* *}(\mathfrak{G})$, it follows from the maximality of $\mathfrak{M}$ that

$$
L^{* *}(\mathfrak{S})=\mathfrak{M}+L^{* *}(\mathfrak{S}) \pi x .
$$


Thus $E=M+A \pi x, M \in \mathfrak{M}$. Since $\pi L(\mathfrak{S})$ is central and $\pi$ is a homomorphism, for any $y \in S_{\theta}$,

$$
\pi y=\pi y E=\pi y M+A \pi(x y) .
$$

Now $\bar{\mu}(y)=0$ for $\bar{\mu} \notin \theta$. Also $\bar{\mu}(x)=0$ for $\bar{\mu} \in \theta$. Hence

$$
\bar{\mu}(x y)=\bar{\mu}(x) \bar{\mu}(y)=0 \quad \text { for all } \quad \mu \in \mathfrak{S S} .
$$

The semi-simplicity of $L(\mathfrak{S})$ yields $x y=0$, and consequently $\pi y=$ $\pi y M \in \mathfrak{M}$, and thus $\mathfrak{M} \supset \pi S_{\theta}$.

Lemma 2.2. If $\mathfrak{M} \supset \pi T(\gamma), \gamma \in \hat{\mathfrak{S}}$, then $\mathfrak{M} \supset \pi \mathfrak{M}_{*_{\gamma}}$.

Proof. Let $x \in \mathfrak{M}_{*}$. Then [7, p. 151] $x=\lim x y_{n}$ with $x y_{n} \in T(\gamma)$. Since $\pi T(\gamma) \subset \mathfrak{M}$ which is norm closed and $\pi$ is continuous, it follows that $\pi x \in \mathfrak{M}$. Thus $\pi \mathfrak{M}_{* \gamma} \subset \mathfrak{M}$.

Theorem 2.3. Let $\mathfrak{M}$ be a maximal modular left ideal in $L^{* *}(\mathfrak{G H})$. Then either $\mathfrak{M} \supset \pi L(\mathbb{S})$ or there exists a unique $\gamma \in \hat{\mathfrak{S} S}$ such that $\mathfrak{M} \supset \pi \mathfrak{M}_{* \gamma}$.

Proof. If $\mathfrak{M} \supset \pi \mathfrak{M}_{*_{\gamma}}$ and $\mathfrak{M} \supset \pi \mathfrak{M}_{*_{\mu}}$ for $\gamma, \mu \in \hat{\mathbb{S}}$ and $\mu \neq \gamma$, then $\mathfrak{M} \supset \pi L(\mathfrak{S})$. Thus we assume that $\mathfrak{M} \nsubseteq \pi \mathfrak{M}_{*_{\gamma}}$ for any $\gamma \in \hat{\mathfrak{S}}$ and show that this leads to a contradiction. It follows from Lemma 2.2 that $\mathfrak{M} \ngtr \pi T(\gamma)$ for any $\gamma \in \hat{\mathfrak{S}}$. Consequently by Lemma 2.1 for each $\gamma \in \hat{\mathfrak{S}}$, there is an open set $\theta(\gamma)$ in $\hat{\mathfrak{S}}$ such that $\mathfrak{M} \supset \pi S_{\theta(\gamma)}$. Let $\mathfrak{\Im}$ be the sum of the ideals $S_{\theta(\gamma)}, \gamma \in \hat{\mathfrak{S}}$, in $L(\mathfrak{S})$, i.e. $\mathfrak{\Im}$ is the smallest ideal in $L(\mathbb{G})$ containing all the $S_{\theta(\gamma)}$. If $\Im$ were not dense in $L(\mathfrak{S})$, then by the Tauberian property of $L(\mathfrak{S})$, there would exist a $\delta \in \hat{\mathfrak{S}}$ such that $\mathfrak{\Im} \subset \mathfrak{M}_{* \delta}$, and consequently $\bar{\delta}(x)=0$ for all $x \in \mathfrak{\Im}$. Since $\mathfrak{\Im} \supset S_{\theta(\delta)}$ the regularity of the Banach algebra $L(\mathrm{G})$ yields an $x \in S_{\theta(\delta)}$ such that $\delta(x)=1$. This contradiction shows that $\mathfrak{I}$ is dense in $L(\mathfrak{S})$ and consequently $\pi \mathfrak{\Im}$ is dense in $\pi L(\mathfrak{S})$. Since $\mathfrak{M} \supset \pi S_{\theta(\gamma)}$ for all $\gamma \in \hat{\mathfrak{S}}, M \supset \pi \mathfrak{F}$. As $\mathfrak{M}$ is closed it follows that $\mathfrak{M} \supset \pi L(\mathfrak{S})$. The conclusion $\mathfrak{M} \supset \pi L(\mathfrak{S})$ is inconsistent with our supposition that $\mathfrak{M} \ngtr \pi \mathfrak{M}_{*_{\gamma}}$ for any $\gamma \in \hat{\mathfrak{S}}$. Consequently the proof of the theorem is complete.

If it were true that $\pi L(\mathbb{S})$ were a two-sided ideal in $\left.L^{* *(B)}\right)$, it would be possible to give a particularly simple algebraic proof of Theorem 2.3 using Theorem 2.6.6 of [9]. However, the next theorem shows that in general the modified argument is not possible since $\pi L(\mathbb{G})$ is ordinarily

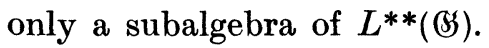

Theorem 2.4. The subalgebra $\pi L(\mathbb{S})$ is a two-sided ideal of $L^{* *(B S)}$ if and only if Bs is compact. 
Proof. Let $\mathfrak{Y}$ denote the smallest closed subspace of $L^{*}(\mathbb{S})$ which contains the multiplicative linear functionals on $L(\mathscr{G S})$, and let $\mathfrak{Y}^{\perp}$ be its linear space annihilator in $L^{* *}(\mathscr{S})$. It is noted in $[6, \mathrm{p} .854]$ that $\mathfrak{Y}^{\perp}$ is

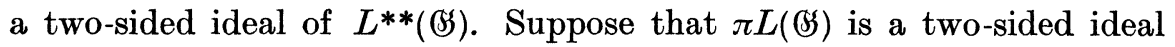
of $L^{* *}(\mathbb{S})$. Let $F \in \mathfrak{Y}^{\perp}$ and $x \in L(\mathbb{S})$. Then $\pi x F \in \pi L(\mathbb{S}) \cap \mathfrak{Y}^{\perp}$. However, if $\pi y \in \pi L(\mathscr{S}) \cap \mathfrak{Y}^{\perp}$, then $\pi y(\mu)=\mu(y)=0$ for all multiplicative linear functionals $\mu$ on $L(G)$. Thus the semi-simplicity of $L(\mathbb{S})$ yields $\pi L(\mathfrak{G S}) \cap \mathfrak{Y}^{\perp}=(0)$. Hence for all $x \in L(\mathfrak{S}), \pi x F=0$. However, since left multiplication in $L^{* *}(\mathbb{G S})$ is $w^{*}$-continuous [2] and since $\pi L(\mathbb{S})$ is $w^{*}$ dense in $L^{* *}(\mathfrak{G S})$ it follows that $L^{* *}(\mathfrak{S}) F=0$ for all $F \in \mathfrak{Y}^{\perp}$. In particular $\mathfrak{Y}^{\perp} \subset \mathfrak{R}^{* *}$, the radical of $L^{* *}(\mathfrak{S})$.

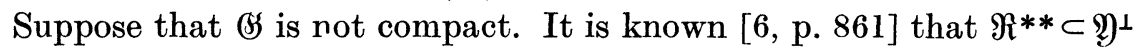
and that the inclusion is proper. Consequently the prior argument shows that if $\pi L(\mathbb{S})$ is a two sided ideal of $L^{* *}(\mathbb{S})$ then $\mathbb{S} 5$ is compact.

Suppose now that $(\mathscr{S}$ is compact. Since $\pi L(\mathbb{S})$ is central, it suffices to see that it is a right ideal. Let $x \in L(\mathfrak{S})$ and let $F \in L^{* *}(\mathbb{S})$. Suppose that $F$ when restricted to the continuous functions corresponds to the countably additive regular Borel measure $\mu$. For any $f \in L^{*}(\mathfrak{F})$, $\pi x F(f)=F(\langle f, x\rangle)$, where the notation is that of [6]. Thus

$$
\pi x F(f)=\int\langle f, x\rangle(t) d \mu(t),
$$

since it was noted in $[6$, p. 856] that $\langle f, x\rangle$ has a representation as a continuous function on $\mathscr{B S}$ with value at $t \in \mathbb{B S}$ equal to $\int f(\alpha) x(\alpha-t) d \alpha$. Then

$$
\pi x F(f)=\int\left\{\int f(\alpha) x(\alpha-t) d \alpha\right\} d \mu(t)=\int\left\{\int x(\alpha-t) d \mu(t)\right\} f(\alpha) d \alpha,
$$

since both measures involved are now countably additive. Let $h(\alpha)=$ $\int x(\alpha-t) d \mu(t)$. Then

$$
\int|h(\alpha)| d \alpha \leqq \iint|x(\alpha-t)| d|\mu|(t) d \alpha \leqq\|x\|\|\mu\|,
$$

where $\|\mu\|$ indicates the usual norm for a countably additive measure. Thus $h \in L(\mathfrak{S})$ and we see that $\pi x F(f)=f(h)=\pi h(f)$ for any $f \in L^{*}(\mathfrak{S})$. Thus $\pi x F=\pi h$ and since $x$ and $F$ were arbitrary in $L(\mathbb{S})$ and $L^{* *}(\mathbb{S})$

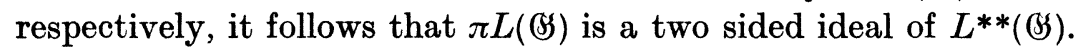

\section{Existence questions.}

From the introduction to Section 2, it can be seen that the two-sided ideal $\mathfrak{M}_{\gamma}, \gamma \in \hat{\mathfrak{S}}$, intersects $\pi L(\mathfrak{S})$ in $\pi \mathfrak{M}_{*_{\gamma}}$. From Theorem 2.3, it follows that any maximal modular left ideal in $L^{* *}(\mathscr{G})$ either contains $\pi L(\mathbb{S})$ or 
intersects it in a set of the form $\pi \mathfrak{M}_{* \gamma}$ for some $\gamma \in \hat{\mathfrak{S}}$. In this section, we consider the question of existence of maximal modular ideals of the former type and of the existence of those of the second type distinct from those of the form of $\mathfrak{M}_{\gamma}$.

Theorem 3.1. There exist maximal left ideals $\mathfrak{M}$ in the algebra $L^{* *}(\mathfrak{S})$, such that $\mathfrak{M} \ngtr \pi L(\mathfrak{G})$ and $\mathfrak{M} \neq \mathfrak{M}_{\gamma}$ for any $\gamma \in \hat{\mathfrak{S}}$ if and only if $\mathfrak{G S}$ is not compact.

Proof. Suppose (S) is not compact. Let $\mathbb{E}$ be the subspace of $L^{*}\left(\bigotimes^{\prime}\right)$ generated by the continuous functions on $\mathbb{E S}$ vanishing at infinity, and

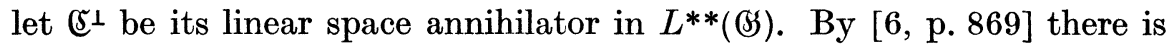
an algebraic isomorphism, $\alpha$, of $L^{* *}(\mathbb{S}) / \mathbb{C}^{\perp}$ onto $M(\mathbb{S})$, the algebra of countably additive regular Borel measures on $\mathbb{S}$. Let $\mathfrak{\Im}_{\gamma}$ be the maximal ideal in $M(\mathscr{B})$ corresponding to $\gamma \in \hat{\mathfrak{S}}$, that is

$$
\Im_{\gamma}=\left\{v \in M\left((\mathscr{S}) \mid \int \bar{\gamma}(t) d v(t)=0\right\} .\right.
$$

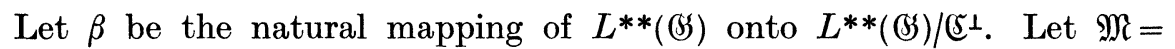
$(\alpha \beta)^{-1} \mathfrak{\Im}_{\gamma}$. Since each of $\alpha$ and $\beta$ is an algebra homomorphism, $\mathfrak{M}$ is a maximal left ideal in $L^{* *}(\mathfrak{S})$ and $\mathfrak{M} \supset \mathfrak{C}^{\perp}$. Suppose that $\mathfrak{M}=\mathfrak{M}_{\delta}$, for some $\delta \in \hat{\mathfrak{S}}$. Then $\mathfrak{M}_{\delta} \supset \mathfrak{C} \mathbb{C}^{\perp}$, so $\mathfrak{M}_{\delta}^{\top} \subset \mathfrak{C}$ and thus $\delta \in \mathfrak{E}$. However, since $\left(\mathfrak{S}\right.$ is not compact, this is a contradiction, and so $\mathfrak{M} \neq \mathfrak{M}_{\delta}$ for any $\delta \in \hat{\mathfrak{S}}$. Let $x \in L(\mathscr{B})$. From the way in which $\alpha$ and $\beta$ are defined (see [6, p. 860]),

$$
\alpha \beta(\pi x)=\alpha\left(\pi x+\mathfrak{\complement}^{\perp}\right)=\pi x \mid \mathfrak{⿷},
$$

the restriction of $\pi x$ to $\mathfrak{E}$. The value of the Fourier transform of $(\pi x \mid \mathfrak{E})$ at $\gamma \in \hat{\mathfrak{S}}$ is given by

$$
\int \bar{\gamma}(t) x(t) d t=\bar{\gamma}(x)=\pi x(\bar{\gamma}) .
$$

Thus $\alpha \beta(\pi x) \in \mathfrak{\Im}_{\gamma}$ if and only if $\pi x \in \mathfrak{M}_{\gamma}$, or if and only if $x \in \mathfrak{M}_{* \gamma}$. Thus $\mathfrak{M} \ngtr \pi L(\mathfrak{S})$, and $\mathfrak{M} \neq \mathfrak{M}_{\gamma}$ for any $\gamma \in \hat{\mathfrak{S}}$, and consequently any maximal left ideal obtained in this fashion is of the required type.

Suppose now that $\mathscr{B S}$ is compact, and suppose that $\mathfrak{M}$ is a maximal left ideal of $L^{* *}(\mathfrak{S})$ such that $\mathfrak{M} \ngtr \pi L(\mathfrak{S})$ and $\mathfrak{M} \supset \pi \mathfrak{M}_{*_{\gamma}}$ for some

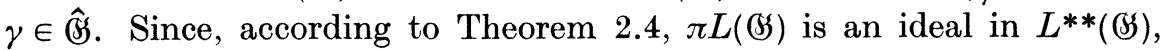
$\mathfrak{M} \cap \pi L(\mathscr{S})=\mathfrak{M}_{\gamma} \cap \pi L(\mathfrak{S})$ is a maximal modular ideal in the algebra $\pi L(\mathfrak{G})$. Let $\pi j$ be an identity modulo the latter ideal in $\pi L(\mathfrak{G})$. Then $\pi j \notin \mathfrak{M}_{\gamma}$, and $\pi j \notin \mathfrak{M}$. It follows that

$$
\pi j^{2}-\pi j \in \mathfrak{M} \cap \pi L(\mathscr{S}),
$$




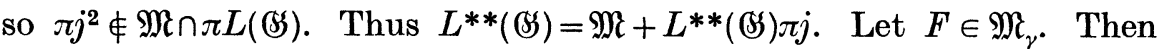
$F=M+Q \pi j$, with $M \in \mathfrak{M}$. Since $\pi j$ is central, $\pi j F=\pi j M+Q \pi j^{2}$. As $\pi j F \in \pi L(\mathbb{S}) \cap \mathfrak{M}$, we see that $Q \pi j^{2} \in \mathfrak{M}$. Since

$$
Q\left(\pi j^{2}-\pi j\right) \in \mathfrak{M} \cap \pi L(\mathscr{S}),
$$

it follows that $Q \pi j \in \mathfrak{M}$ and thus $F \in \mathfrak{M}$. We therefore conclude that $\mathfrak{M}=\mathfrak{M}_{\gamma}$.

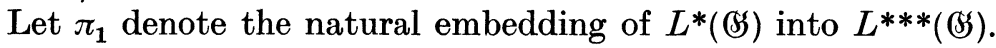

Corollary 3.2. If (S) is not compact there exist multiplicative linear functionals on $L^{* *(\mathscr{S})}$ which are not of the form $\pi_{1} \gamma, \gamma \in \hat{\mathscr{S}}$.

Proof. Let the notation be as in the first part of the proof of Theorem 3.1. Let $\varphi \in(M(\mathfrak{S}))^{*}$ be the multiplicative linear functional corresponding to the evaluation of the Fourier transform at $\gamma \in \hat{\mathfrak{S}}$. Let $\psi=(\alpha \beta)^{*}(\varphi)$,

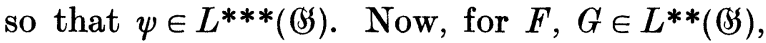

$$
\psi(F G)=(\alpha \beta)^{*}(\varphi)(F G)=\varphi(\alpha \beta(F G))=\varphi(\alpha \beta(F)) \varphi(\alpha \beta(G))=\psi(F) \psi(G),
$$

so $\psi$ is multiplicative, and is clearly not the zero functional. Thus the

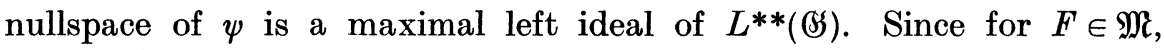
$\psi(F)=\varphi(\alpha \beta(F))=0$, it follows that the nullspace of $\psi$ is $\mathfrak{M}$. Now if $\psi=\pi_{1} \delta$ for some $\delta \in \hat{\mathfrak{B}}$, then $\mathfrak{M}=\mathfrak{M}_{\delta}$ in contrast to what was observed in the proof of Theorem 3.1.

Theorem 3.3. If BS is not discrete, there exists a maximal left ideal $\mathfrak{M}$ in $L^{* *}(\mathfrak{S})$ such that $\mathfrak{M} \supset \pi L(\mathbb{S})$.

Proof. If $(B)$ is compact, then Theorem 2.2 asserts that $\pi L(\mathbb{F})$ is an ideal. Since $L^{* *}(\mathfrak{S})$ has a right identity $E, \pi L(\mathfrak{S})$ is a proper modular left ideal and the existence of the asserted ideal is immediate.

If $\mathfrak{B S}$ is not compact, consider the ideal $\mathfrak{\Im}$ in $L^{* *}(\mathfrak{S})$ generated by $\pi L(\mathbb{S})$. By the argument used above, it suffices if we show $E \notin \Im$. Suppose the contrary. Then there exist $x_{i} \in L(\mathbb{S})$ and $F_{i} \in L^{* *}(\mathbb{S})$, $i=1, \ldots, n$, such that $E=\sum_{i=1}^{n}\left(\pi x_{i}\right) F_{i}$. Thus for any $y \in L(\mathscr{S}), \pi y=$ $\sum_{i=1}^{n}\left(\pi\left(y x_{i}\right)\right) F_{i}$. Since $\pi L(\mathbb{B S})$ is central in $L^{* *}(\mathfrak{B S})$, it follows that for any $W \in L^{* *}(\mathbb{S})$,

$$
\sum_{i=1}^{n}\left(\pi\left(y x_{i}\right)\right)\left(W F_{i}-F_{i} W\right)=0 .
$$

From the $w^{*}$-density of $\pi L(\mathscr{B S})$ in $L^{* *}(\mathbb{G})$, we see that there is a net $\left\{\pi y_{\alpha}\right\}$ which is $w^{*}$-convergent to $E$. The $w^{*}$-continuity of left multiplication together with relation (4) then yields 
$0=E \sum_{i=1}^{n} \pi x_{i}\left(W F_{i}-F_{i} W\right)=\sum_{i=1}^{n} \pi x_{i}\left(W F_{i}-F_{i} W\right), \quad$ or $\quad W E-E W=0$.

This asserts that $W=E W$, for all $W \in L^{* *((S)) . ~ H o w e v e r, ~ f o r ~(B) ~ n o t ~}$ discrete, $L^{* *}(\mathbb{( G )})$ has no identity since $L^{* *}(\mathbb{S})$ has $[6$, p. 856] a non-zero right annihilator.

It should be noted that for (S) discrete the prior result clearly fails since $L(\mathscr{G S})$ and therefore $[6, \mathrm{p} .865] L^{* *}(\mathfrak{S S})$ has an identity.

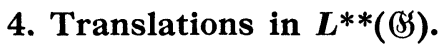

Let $T_{\lambda}, \lambda \in \mathbb{S}$, denote the translation operator in $L(\mathbb{S})$, that is, for $x \in L(\mathfrak{S}), \quad T_{\lambda} x(\beta)=x(\lambda+\beta), \quad \beta \in(\mathfrak{S S}$. It is a well known phenomenon [7, p. 125] that a closed subspace in $L(\mathscr{S})$ is an ideal if and only if it is invariant under each $T_{\lambda}, \lambda \in \mathbb{B S}$. The object of the present section is to discuss the similarities and differences in this type of behavior where one considers translations in $L^{* *}(\mathbb{S})$.

It is immediately verifiable that when an element $F$ of $L^{* *}(\mathscr{S})$ is interpreted as a finitely additive set function, the set function corresponding to the translation of the original by $\lambda \in \mathbb{B S}$ comes from operation on $F$ by $T_{\lambda}{ }^{* *}$. Thus we are concerned with questions of invariance of subspaces of $L^{* *}(\mathbb{S})$ under $T_{\lambda}{ }^{* *}$. The notation which will be used is that of [6].

Lemma 4.1. For any $\lambda \in \mathbb{B S}, T_{\lambda}{ }^{* *}(F G)=F\left(T_{\lambda}{ }^{* *} G\right)=\left(T_{\lambda}{ }^{* *} F\right) G$.

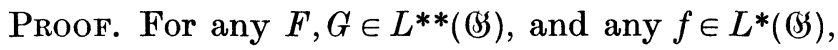

$$
T_{\lambda}{ }^{* *}(F G)(f)=F G\left(T_{\lambda}^{*} f\right)=F\left(\left[G, T_{\lambda}{ }^{*} f\right]\right) .
$$

Also for each $x \in L(\mathfrak{G}),\left[G, T_{\lambda}{ }^{*} f\right](x)=G\left(\left\langle T_{\lambda}{ }^{*} f, x\right\rangle\right)$. But for each $y \in L(\mathfrak{G})$,

$$
\left\langle T_{\lambda}{ }^{*} f, x\right\rangle(y)=T_{\lambda}{ }^{*} f(x y)=f\left(T_{\lambda}(x y)\right)=f\left(\left(T_{\lambda} x\right) y\right)=f\left(x\left(T_{\lambda} y\right)\right) .
$$

If the first interpretation is used, we conclude $\left\langle f, T_{\lambda} x\right\rangle(y)=\left\langle T_{\lambda}{ }^{*} f, x\right\rangle(y)$ for all $y \in L(\mathbb{S})$ and thus $\left\langle f, T_{\lambda} x\right\rangle=\left\langle T_{\lambda}{ }^{*} f, x\right\rangle$. Thus

$$
\left[G, T_{\lambda}^{*} f\right](x)=G\left(\left\langle f, T_{\lambda} x\right\rangle\right)=[G, f]\left(T_{\lambda} x\right)=T_{\lambda}{ }^{*}[G, f](x)
$$

for all $x \in L(\mathbb{S})$. Consequently $\left[G, T_{\lambda}{ }^{*} f\right]=T_{\lambda}{ }^{*}[G, f]$ and

$$
T_{\lambda}^{* *}(F G)(f)=F\left(T_{\lambda}^{*}[G, f]\right)=\left(T_{\lambda}^{* *} F\right) G(f) .
$$

Hence $T_{\lambda}{ }^{* *}(F G)=\left(T_{\lambda}{ }^{* *} F\right) G$. If the second interpretation were used above, a like calculation would show $T_{\lambda}{ }^{* *}(F G)=F\left(T_{\lambda}{ }^{* *} G\right)$. 
Theorem 4.2. If $\mathfrak{\Im}$ is a right ideal in $L^{* *((\Im)), ~ o r ~ i f ~} \mathfrak{\Im}$ is a left ideal that contains the right annihilator of $L^{* *}(\mathbb{S})$, then $\mathfrak{\Im}$ is invariant under $T_{\lambda}{ }^{* *}$, $\lambda \in \mathfrak{G}$.

Proof. The condition that a left ideal contain the right annihilator is, of course, automatically satisfied if $\mathscr{B S}$ is discrete since in this case the right annihilator is zero. However, it will be shown later that for (5) not discrete, the condition is essential.

Suppose first that $\Im$ is a right ideal and $F \in \mathfrak{~}$. Then $F=F E$, where $E$

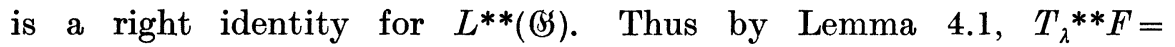
$F\left(T_{\lambda}{ }^{* *} E\right) \in \mathfrak{\Im}$. If $\mathfrak{\Im}$ is a left ideal and $F \in \mathfrak{\Im}$, then $E F \in \mathfrak{\Im}$, where $E$ is as above. Thus

$$
T_{\lambda}^{* *}(E F)=E\left(T_{\lambda}^{* * F}\right)=\left(T_{\lambda}{ }^{* *} E\right) F \in \mathfrak{\Im} .
$$

Since $E\left(T_{\lambda}{ }^{* *} F\right)-\left(T_{\lambda}{ }^{* *} F\right)$ is automatically in the right annihilator of

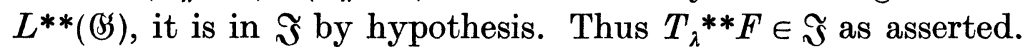

Before proceeding to the demonstration that the condition on the right annihilator is essential, it is necessary to establish an intermediate result, which was used without proof in $[6$, p. 856] for the special case $c=0$.

Lemma 4.3. If (S) is not discrete, then there exists $f \in L^{*}(\mathbb{S})$ and $\lambda \in \mathbb{B S}$ such that $f-c T_{\lambda}{ }^{*} f$ has no continuous representation for any complex $c$.

Proof. Let $\lambda \neq 0, \lambda \in \mathbb{B S}$. Let $W$ be a neighborhood of 0 with $\bar{W}$ compact such that $\bar{W} \cap(-\lambda+\bar{W})=0$. We shall show below that there exist $f \in L^{*}(\mathfrak{G S})$ such that no function equal to $f$ in the sense of $L^{*}(\mathfrak{G})$ is continuous and such that $\{t \mid f(t) \neq 0\} \subset W$. Suppose some function $f^{\prime}$ equal to $f$ in $L^{*}(\mathscr{G})$ satisfies $f^{\prime}-c T_{\lambda}{ }^{*} f^{\prime}=g$, with $g$ continuous, and $c$ a complex number. Then for $t \in W, f(t)=g(t)$ which is in contradiction to the supposed nature of $f$. The proof thus reduces to the construction of the desired $f$.

Suppose that there exists an open set $U, \bar{U} \subset W$ and such that $\bar{U}$ is not open. Let $f(t)=1, t \in U$ and $f(t)=0, t \notin U$. Since $\bar{U}$ is compact, $\bar{U}-U$ is compact and $U$ is measurable and thus $f \in L^{*}(\mathfrak{G})$. Let $t_{0} \in \bar{U}-U$ be such that no neighborhood of $t_{0}$ is contained in $\bar{U}$. Since any open set containing $t_{0}$ contains points both of $U$ and the complement of $\bar{U}$, the alteration of $f$ on a set of measure zero will not alter the discontinuity at $t_{0}$.

Suppose next that each open set $V$ with $\bar{V} \subset W$ has $\bar{V}$ open. Let $U$ be open, $0 \in U$ and $\bar{U} \subset W$. Then $[8$, p. 54] there is a compact open subgroup $\mathfrak{H}$ of $\mathfrak{S S}$ with $\mathfrak{S} \subset \bar{U}$. Clearly $\mathfrak{H}$ is not discrete. If the desired function is constructed for the compact group $\mathfrak{B}$, its extension to $\mathfrak{B S}$ by defining it to be zero off $\mathfrak{g}$ would clearly have the desired property. 
In view of the earlier argument, we may suppose that each open set in $\mathfrak{H}$ has an open closure. Since $0 \in \mathfrak{F}$ is the limit of a net $\left\{x_{\alpha}\right\}$ in $\mathfrak{G}$ with 0 deleted, there exists an infinite collection of disjoint compact open sets $V_{n}$ in $\mathfrak{W}$, such that each $V_{n}$ contains some $x_{\alpha}$. Let $f(t)=n^{-1}$ for $t \in V_{n}$, and $f(t)=0$ for $t \notin \cup V_{n}$. The function $f$ is then continuous real valued, and its range is an infinite set. By [8, p. 60] for any such $g$, there is a compact subgroup $\mathfrak{G}_{1}$ of $\mathfrak{S}$ and such that $\mathfrak{S} / \mathfrak{F}_{1}$ is separable metric and $g$ is constant on cosets modulo $\mathfrak{G}_{1}$. Since the range of $g$ is infinite, $\mathfrak{\mathfrak { C }} / \mathfrak{G}_{1}$ is an infinite group and since it is compact cannot be discrete. Since $\mathfrak{S} / \mathfrak{H}_{1}$ is separable metric, there exist open sets $U_{n}$ and $V_{n}$ in $\mathfrak{S} / \mathfrak{S}_{1}$ such that $x_{n} \in U_{n}$ implies $\lim x_{n}=0$ in $\mathfrak{S} / \mathfrak{Q}_{1}$ and $y_{n} \in V_{n}$ implies $\lim y_{n}=0$ in $\mathfrak{S} / \mathfrak{H}_{1}$. The sets $U_{n}$ and $V_{n}$ may also be taken so that $\bar{U}_{n} \cap V_{j}=\varnothing$ for all $n$ and $j$, and also $0 \notin \bar{U}_{n} \cup \bar{V}_{n}$ for any $n$. Let $\alpha$ denote the natural mapping from $\mathfrak{S}$ onto $\mathfrak{S} / \mathfrak{S}_{1}$, and let

$$
X_{n}=\alpha^{-1} U_{n}, \quad Y_{n}=\alpha^{-1} V_{n} .
$$

Let $f(t)=1, t \in X_{n}, n=1,2, \ldots$, and $f(t)=0$ otherwise and in particular on each $Y_{n}$. Clearly $f \in L^{*}(\mathfrak{S})$. Let $x_{0} \in \mathfrak{S}_{1}$. Let $Z$ be an open set in $\mathfrak{S}$ which contains $x_{0}$. Then $\alpha Z$ is open and there is a $u_{n} \in \alpha Z \cap U_{n}$, and a $v_{n} \in \alpha Z \cap V_{m}$ for some integers $n$ and $m$. Since $f$ is constant on cosets $\bmod \mathfrak{H}_{1}$, there exist open sets in $Z \cap X_{n}$ where $f$ has the value 1 and open sets in $Z \cap Y_{m}$ where $f$ has the value 0 . Consequently $f$ cannot be modified on a set of measure zero so as to become continuous and the proof is complete.

Theorem 4.4. If (5) is not discrete, there exists a closed left ideal in $L^{* *}(\mathbb{S})$ which is not invariant under $T_{\lambda}^{* *}, \lambda \in \mathfrak{G S}$.

Proof. Let $f \in L^{*}(\mathbb{S}), \lambda \in \mathbb{S}$ be such that $f-a T_{\lambda}{ }^{*} f$ has no continuous representative in $L^{*}(\mathbb{G})$ for any complex $a$. Let $\Im_{f}$ be the closed subspace of $L^{*}(\mathbb{S})$ spanned by $f$ and

$$
I=\left\{\langle g, x\rangle \mid g \in L^{*}(\mathfrak{G}), x \in L((\mathfrak{S})\} .\right.
$$

Suppose that $T_{\lambda}{ }^{*} f \in \Im_{f}$.

Then there exist $g_{n} \in \mathfrak{I}$ and $a_{n}$ complex, $n=1,2, \ldots$, such that $\lim \left(g_{n}+a_{n} f\right)=T_{\lambda} * f$. Suppose that $\left\{a_{n}\right\}$ were bounded. Let $\left\{b_{m}\right\}$ be a subsequence of $\left\{a_{n}\right\}$ with $\lim b_{m}=b$. We would then have

$$
\lim \left(g_{m}{ }^{\prime}+b_{m} f\right)=\lim g_{m}{ }^{\prime}+b f=T_{\lambda}{ }^{*} f,
$$

with each $g_{m}{ }^{\prime} \in \Im$. Since $[6$, p. 856] each of the elements $\langle g, x\rangle$ spanning $\Im$ is continuous and convergence is uniform, we deduce that $T_{\lambda}{ }^{*} f-b f$ 
is continuous. Thus $\left\{a_{n}\right\}$ cannot be bounded. We may, without loss of generality, assume that $\left\{a_{n}\right\}$ tends to infinity. Then

$$
\lim \left(a_{n}{ }^{-1} g_{n}+f\right)=\lim a_{n}{ }^{-1} T_{\lambda}{ }^{*} f=0,
$$

and we deduce that $f$ is continuous, contrary to our selection of $f \in L^{*}(\mathbb{( S )})$. Consequently we deduce that $T_{\lambda}{ }^{*} f \notin \Im_{f}$.

By the Hahn-Banach theorem, there is a functional $F \in L^{* *}(\mathfrak{S})$ such that $F(g)=0$ for $g \in \Im_{f}$ and $F\left(T_{\lambda}{ }^{*} f\right)=1$. It follows from [6, p. 855] that $F$ is in the right annihilator of $L^{* *}(\mathscr{S})$. Since $T_{\lambda}{ }^{* *} F(f)=F\left(T_{\lambda}{ }^{*} f\right)=1$, while $F(f)=0$, it also follows that $T_{\lambda}^{* * F} \neq c F$ for any complex $c \neq 0$. As $T_{\lambda}{ }^{* *} F=0$ implies $F=0$, we conclude $T_{\lambda}{ }^{* *} F \neq c F$ for any complex $c$.

Let $\mathfrak{R}=\{c F \mid c$ complex $\}$. Since $F$ is in the right annihilator of $L^{* *}(\mathfrak{S})$, $\mathfrak{R}$ is a left ideal of $L^{* *}(\mathfrak{G})$. Also from $T_{\lambda}{ }^{* *} F \neq c F$ for any complex $c$, we see that $\mathfrak{R}$ is not invariant under all $T_{\mu}{ }^{* *}, \mu \in \mathbb{B}$.

We next address the question of whether a closed subspace of $L^{* *}(\mathbb{S})$, invariant under $T_{\lambda}{ }^{* *}, \lambda \in \mathbb{S S}$, is a left or right ideal. The answer is clearly negative if $\mathscr{B S}$ is not compact, for $\pi L(\mathscr{B S})$ is clearly invariant under $T_{\lambda}{ }^{* *}$ for each $\lambda \in \mathbb{B S}$ and is not an ideal by Theorem 2.4. The answer is also negative if $\mathfrak{G S}$ is compact. It is convenient to prove an intermediate result with regard to translation invariance in $M(\mathbb{S})$.

Theorem 4.5. If (BS is not discrete, there exists a closed subspace of $M\left(\mathbb{S}_{)}\right)$which is invariant under translation and which is not an ideal in $M(\mathfrak{S})$.

Proof. Let $\mathfrak{I}$ denote the closed subspace of $M(\mathfrak{S})$ generated by the point masses. Clearly $\mathfrak{I}$ is invariant under translation. Suppose that $\mathfrak{I}$ is an ideal in $M(\mathfrak{S})$. Let $\beta$ denote the natural embedding of $L(\mathfrak{B S})$ into $M(\mathbb{G S})$. It follows $[3$, p. 54] that there is a continuous projection $p$ of norm one of $M(\mathbb{S})$ onto $\beta L(\mathbb{S})$ such that for each $\mu \in M(\mathbb{S})$,

$$
\|\mu\|=\|p(\mu)\|+\|\mu-p(\mu)\| .
$$

Furthermore, the kernel of $p$ is the set of measures on $(S)$ singular with respect to Haar measure. In particular, $\mathfrak{F} \subset p^{-1}(0)$. Since $\beta L(\mathfrak{B S})$ is an ideal in $M(\mathscr{S})$, it follows that for each $x \in L(\mathscr{S})$ and $\mu \in \mathfrak{F}$,

$$
\beta x * \mu \in \mathfrak{I} \cap \beta L(\mathscr{S}) \subset p^{-1}(0) \cap \beta L(\mathbb{S}) .
$$

Since $p$ is a projection onto $\beta L(\mathbb{S})$ it follows that $\beta x * \mu=p(\beta x * \mu)=0$. As the latter relation holds for each point mass $\mu$, we deduce that $\beta x=0$ and $x=0$ which contradicts the arbitrary nature of $x \in L(\mathbb{S})$. Therefore $\mathfrak{I}$ is not an ideal in $M(\mathbb{S})$. 
Theorem 4.6. There exists a closed subspace of $L^{* *(\mathscr{S})}$ which is invariant under $T_{\lambda}{ }^{* *}$ for all $\lambda \in \mathscr{G S}$ and which is neither a left nor a right ideal.

Proof. In view of the comments made prior to Theorem 4.5, we may suppose that $\mathscr{H}$ is compact and since $(\mathfrak{S})$ is infinite we may suppose $\mathscr{G}$ is not discrete. If $\mathbb{E}$ denotes the subspace of $L^{*}(\mathfrak{S S})$ consisting of the continuous functions on $\mathbb{G S}$, then $\mathbb{C}^{\perp}$ is an ideal of $L^{* *}(\mathbb{S})$ and $L^{* *}(\mathbb{S}) / \mathbb{C}^{\perp}$ is isomorphic $[6$, p. 859] as an algebra to $M(\mathbb{G})$. Consequently there exists an algebra homomorphism, $\varrho$, of $L^{* *}(\mathfrak{S})$ onto $M(\mathfrak{G S})$ which is definable in a natural way and which carries the embedding of $L(\mathscr{H})$ in $L^{* *}(\mathfrak{S})$ onto the embedding of $L(\mathfrak{S})$ in $M(\mathfrak{S})$. Let $\mathfrak{\Im}$ be the subspace of $M(\mathscr{G})$ that is used in the proof of Theorem 4.5. Then $\varrho^{-1} \Im$ is a closed subspace of $L^{* *((5)}$ which is neither a left nor a right ideal. Furthermore the invariance of $\mathfrak{\Im}$ under translation implies the invariance of $\varrho^{-1} \mathfrak{\Im}$ under $T_{\lambda}{ }^{* *}$ for all $\lambda \in \mathfrak{W}$. Consequently $\varrho^{-1} \mathfrak{\Im}$ is a subspace of the required type.

A slightly more analogous structure is obtained for $L(\mathfrak{B S})$ and $L^{* *}(\mathfrak{S})$ if the translation operator $T$ on $L(\mathbb{S S})$ is replaced by a related operator.

For $K$ a compact neighborhood in $\mathbb{B r}$, let $T_{K}$ be defined on $L(\mathbb{S})$ by

$$
T_{K} x(\gamma)=\int x(\gamma+t) \varphi_{K}(t) d t, \quad x \in L(\mathbb{S}), \gamma \in \mathbb{S},
$$

where $\varphi_{K}$ is the characteristic function of the set $K$. Since

$$
\begin{aligned}
\int\left|T_{K} x(\gamma)\right| d \gamma & =\int\left|\int x(\gamma+t) \varphi_{K}(t) d t\right| d \gamma \\
& \leqq \iint|x(\gamma+t)| d \gamma \varphi_{F}(t) d t=m(K)\|x\|,
\end{aligned}
$$

it follows that $T_{K}$ is a bounded operator on $L(\mathbb{S})$.

Lemma 4.7. For $x, y \in L(\mathfrak{F}), T_{K}(x y)=x T_{K} y$.

Proof. This follows by direct computation.

Theorem 4.8. A closed subspace $\mathfrak{\Im}$ of $L(\mathscr{S})$ is an ideal if and only if it is invariant under $T_{K}$ for each compact neighborhood $K$.

Proof. If $\mathfrak{\Im}$ is a closed ideal, the use of an approximate identity together with Lemma 4.7 yields the invariance of $\mathfrak{\Im}$ under $T_{K}$.

Suppose that $\mathfrak{\Im}$ is a closed subspace of $L(\mathfrak{H})$ invariant under each $T_{K}$. Let $x \in \mathfrak{\Im}$ and let $y_{K}$ be the characteristic function of a compact neighborhood $K$. Then for $\gamma \in \mathbb{B S}$,

$$
x y_{K}(\gamma)=\int x(\gamma-t) y_{K}(t) d t=\int x(\gamma+t) y_{-K}(t) d t=T_{-K} x(\gamma) .
$$


Thus $x y_{K}=T_{-K} x \in I$. Since the linear combinations of the $y_{K}$ are dense in $L(\mathscr{S})$ and $\mathfrak{\Im}$ is closed, it is immediate that $\mathfrak{\Im}$ is an ideal.

In view of Lemma 4.1 the following result can be established in an identical fashion to that of Theorem 4.2.

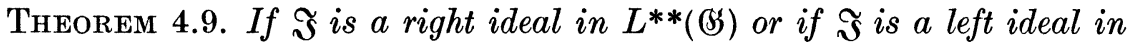

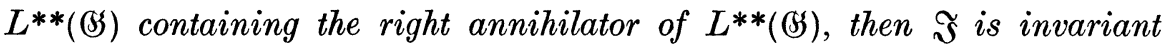
under $T_{K}{ }^{* *}$ for each compact neighborhood $K$ in $\mathfrak{B S}$.

The positive result relating to invariance under $T_{K}{ }^{* *}$ is the following.

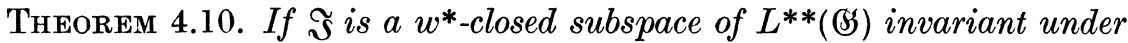
all $T_{K}{ }^{* *}$, then $\mathfrak{\Im}$ is a left ideal.

Proof. Let $\Im^{\top}$ be the linear space annihilator in $L^{*}(\mathfrak{S})$ of $\Im$. Let $f \in \mathfrak{F}^{\top}$ and let $x_{K}$ be the characteristic function of a compact neighborhood $K$. Then the value of $\left\langle f, x_{K}\right\rangle$ at $\gamma \in \mathbb{S}$ is given $[6, \mathrm{p} 856]$ by

$$
\left\langle f, x_{K}\right\rangle(\gamma)=\int f(\gamma+t) x_{K}(t) d t=T_{-K} * f(\gamma),
$$

and so $\left\langle f, x_{K}\right\rangle=T_{-K}^{*} f$. Thus for any $F \in \mathfrak{\Im}$,

$$
F\left(\left\langle f, x_{K}\right\rangle\right)=F\left(T_{-K}^{*} f\right)=T_{-K}^{* *} F(f)=0,
$$

and consequently $\left\langle f, x_{K}\right\rangle \in \Im^{\top}$ for each compact neighborhood $K$. Since the linear combinations of such $x_{K}$ are dense in $L(\mathfrak{S}),\langle f, x\rangle \in \mathfrak{F}^{\top}$ for all $x \in L(\mathfrak{S})$. This, however, is sufficient to show that $\mathfrak{\Im}$ is a left ideal. (See $[4$, p. 1226] where the argument is given for two-sided ideals.)

\section{Linear space annihilators.}

The object in this section is to obtain some information concerning the maximal left ideals in $L^{* *}(\mathbb{S})$ by considering their linear space annihilators. We use the notation introduced in Section 2. The symbols $E$

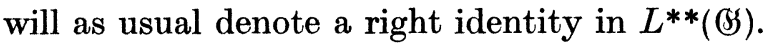

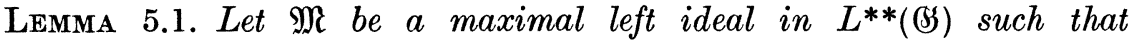
$\mathfrak{M} \ngtr \pi L(\mathfrak{S}), \mathfrak{M} \supset \pi \mathfrak{M}_{* \gamma}$ for some $\gamma \in \hat{\mathfrak{S}}$, but $\mathfrak{M} \neq \mathfrak{M}_{\gamma}$. Let $j$ be an identity modulo $\mathfrak{M}_{*, \cdot}$. Then for any $\varphi \in \mathfrak{M}^{\perp}$

(i) $\varphi=\langle\varphi, \pi j\rangle$,

(ii) $\pi j$ is an identity modulo $\mathfrak{M}$, and

(iii) $\pi j-E \in \mathfrak{M}_{\mathrm{i}} \cap \mathfrak{M}_{\gamma}$.

Proof. For any $F \in L^{* *}(\mathfrak{S})$ and $x \in L(\mathfrak{S})$,

$$
\langle\varphi, \pi j\rangle(\pi x F)-\varphi(\pi x F)=\varphi(\pi(j x-x) F)=\varphi(F \pi(j x-x))=0,
$$


since $\pi L(\mathscr{S})$ is central, $j$ is an identity modulo $\mathfrak{M}_{*_{\gamma}}$ and $\pi \mathfrak{M}_{* \gamma} \subset \mathfrak{M}$. Thus

$$
\varphi-\langle\varphi, \pi j\rangle \in\left(\pi L(\mathbb{S}) \cdot L^{* *}(\mathbb{S})\right)^{\perp} .
$$

Now, $\pi L(\mathfrak{G}) \cdot L^{* *}(\mathfrak{S})$ is a two-sided ideal not contained in $\mathfrak{M}$, since $E \in L^{* *}(\mathfrak{S})$ and $\pi L(\mathfrak{S}) \notin \mathfrak{M}$. Thus

Since

$$
L^{* *}(\mathfrak{G S})=\pi L(\mathfrak{G}) \cdot L^{* *}(\mathfrak{G})+\mathfrak{M} .
$$

$$
\varphi-\langle\varphi, \pi j\rangle \in\left(\pi L(\mathfrak{G}) \cdot L^{* *}(\mathfrak{G})\right)^{\perp} \quad \text { and } \quad \varphi-\langle\varphi, \pi j\rangle \in \mathfrak{M}^{\perp}
$$

it follows that $\varphi=\langle\varphi, \pi j\rangle$.

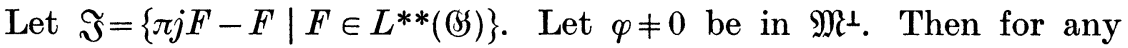
$F \in L^{* *}(\mathfrak{S})$,

$$
\varphi(\pi j F-F)=\langle\varphi, \pi j\rangle(F)-\varphi(F)=0,
$$

by the above. Thus $\varphi \in \mathfrak{I}^{\perp}$ and therefore $\mathfrak{M}^{\perp} \subset \mathfrak{I}^{\perp}$, which implies $\mathfrak{\Im} \subset \mathfrak{M}$. Thus $\pi j$ is an identity modulo $\mathfrak{M}$. Note that by the $w^{*}$-closure of $\mathfrak{M}_{\gamma}$, one also has $\mathfrak{\Im} \subset \mathfrak{M}_{\gamma}$. The last statement then follows by letting $F=E$.

Theorem 5.2. Let $\mathfrak{M}$ be a maximal left ideal in $L^{* *(\mathfrak{S})}$ such that $\mathfrak{M} \ngtr \pi L(\mathfrak{S}), \mathfrak{M} \supset \pi \mathfrak{M}_{*_{\gamma}}$ for some $\gamma \in \hat{\mathfrak{O}}$. Then for any $\varphi \in \mathfrak{M}^{\perp}, x \in L(\mathfrak{S})$ and $F \in L^{* *}(\mathbb{H})$,

$$
\varphi(\pi x F)=\bar{\gamma}(x) \varphi(F) .
$$

Proof. Let $x \in L(\mathbb{S})$. Then if $j$ is an identity modulo $\mathfrak{M}_{\boldsymbol{*}_{\gamma}}, \bar{\gamma}(j)=1$ and $\pi x-\bar{\gamma}(x) \pi j \in \mathfrak{M}_{\gamma}$. Since $\mathfrak{M}_{\cap} \cap \pi L(\mathfrak{G})=\mathfrak{M}_{\gamma} \cap \pi L(\mathscr{G})$, we see that $\pi x-\bar{\gamma}(x) \pi j \in \mathfrak{M}$. Thus for all $G \in L^{* *}(\mathfrak{S})$,

Consequently

$$
(\pi x-\bar{\gamma}(x) \pi j) G=G(\pi x-\bar{\gamma}(x) \pi j) \in \mathfrak{M} .
$$

$$
\varphi(\pi x G)=\varphi(\bar{\gamma}(x) \pi j G)=\bar{\gamma}(x)\langle\varphi, \pi j\rangle(G)=\bar{\gamma}(x) \varphi(G),
$$

which is the desired relation.

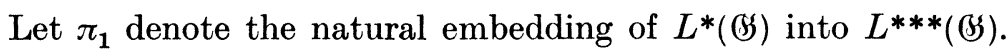

Theorem 5.3. Let $\mathfrak{M}$ be a maximal left ideal in $L^{* *}(\mathfrak{B})$. Suppose that there exist $\varphi \neq 0$, and $\varphi \in \mathfrak{M}^{\perp} \cap \pi_{1} L^{*}(\mathfrak{G S})$. Then $\mathfrak{M}=\mathfrak{M}_{\gamma}$ for some $\gamma \in \hat{\mathfrak{G}}$.

Proof. Say $\varphi=\pi_{1} f, 0 \neq f \in L^{*}(\mathfrak{G})$. If $\mathfrak{M} \supset \pi L(\mathfrak{S})$ then $0=\varphi(\pi x)=f(x)$ for all $x \in L(\mathfrak{G S})$ and so $f=0$. Consequently $\mathfrak{M} \ngtr \pi L(\mathfrak{G S})$. As a consequence of Theorem 2.3, there exists $\gamma \in \hat{\mathfrak{G}}$ such that $\mathfrak{M} \supset \pi \mathfrak{M}_{\boldsymbol{*}_{\gamma}}$. Thus for all $x \in \mathfrak{M}_{* \gamma}, 0=\varphi(\pi x)=f(x)$, but $f(x) \neq 0$ for all $x \in L(\mathfrak{S})$. It follows that $f=c \bar{\gamma}$ for some complex $c \neq 0$. Hence for all $F \in \mathfrak{M}, 0=\varphi(F)=$ $F(c \bar{\gamma})$. Consequently $\mathfrak{M} \subset \mathfrak{M}_{\gamma}$ and thus $\mathfrak{M}=\mathfrak{M}_{\gamma}$. 
Corollary 5.4. All maximal left ideals of $L^{* *((S))}$ other than the $\mathfrak{M}_{\gamma}, \gamma \in \hat{\mathfrak{G}}$, are $w^{*}$-dense.

Proof. The $w^{*}$-closure of $\mathfrak{M}$ is $\mathfrak{M}^{\top \perp}$ and is [5, Lemma 4.7] a left ideal containing $\mathfrak{M}$. Consequently $\mathfrak{M}$ is $w^{*}$-dense unless $\mathfrak{M}=\mathfrak{M}^{\top \perp}$. In this case if $f \neq 0, f \in \mathfrak{M}^{\top}$, then $\pi_{1} f \in \mathfrak{M}^{\perp} \cap \pi_{1} L^{*}(\mathfrak{S})$, and thus $\mathfrak{M}=\mathfrak{M}_{\gamma}$, follows from Theorem 5.3.

It should be noted that the use of Corollary 5.4 affords an opportunity to greatly simplify the arguments in [5] relating to right algebraic annihilators of maximal left ideals.

\section{REFERENCES}

1. R. Arens, Operations induced in function classes, Monatsh. Math. 55 (1951), 1-19.

2. R. Arens, The adjoint of a bilinear operation, Proc. Amer. Math. Soc. 2 (1951), 839-848.

3. N. Bourbaki, Eléménts de mathématique, XXI, Intégration des measures, Paris, 1956.

4. P. Civin, Extensions of homomorphisms, Pacific J. Math. 11 (1961), 1223-1233.

5. P. Civin, Annihilators in the second coniugate algebra of a group algebra, Pacific J. Math. 12 (1962), 855-862.

6. P. Civin and $\mathrm{B}$. Yood, The second conjugate space of a Banach algebra as an algebra, Pacific J. Math. 11 (1961), 847-870.

7. L. H. Loomis, An introduction to abstract harmonic analysis, New York, 1953.

8. D. Montgomery and L. Zippin, Topological transformation groups, New York, 1955.

9. C. E. Rickart, General theory of Banach algebras, New York, 1960.

UNIVERSITY OF COPENHAGEN, DENMARK

AND

UNIVERSITY OF OREGON, EUGENE, OREG., U.S.A. 